\section{A non-specific biomarker of disease activity in HIV/AIDS patients from}

\section{resource-limited environments}

Priyesh Bipath ${ }^{1}$, Peter Levay ${ }^{2}$, Steve Olorunju ${ }^{3}$, Margaretha Viljoen ${ }^{4}$

1. Department of Physiology, School of Medicine, Faculty of Health Sciences, University of Pretoria, South Africa

2. Department of Internal Medicine (Kalafong Hospital), School of Medicine, Faculty of Health Sciences, University of Pretoria, South Africa

3. Biostatistics Unit at Pretoria, Medical Research Council of South Africa

4. Department of Psychiatry, School of Medicine, Faculty of Health Sciences,

University of Pretoria, South Africa

\section{Abstract:}

Background: A general non-specific marker of disease activity that could alert the clinician and prompt further investigation would be of value in patients with HIV/AIDS, especially in resource limited environments.

Objective: To investigate the potential of neopterin as non-specific biomarker in patients with advanced HIV/AIDS Methods: Cross-sectional study in 105 HIV positive patients ( 75 on highly active antiretroviral treatment (HAART). Neopterin was assessed by enzyme linked immune-absorbent assay and cytokines by flow cytometry.

Results: Neopterin levels were significantly higher $(\mathrm{p}<0.001)$ for the total patient than for the control group. Significant Results: Neoptein levels we control group. Significan

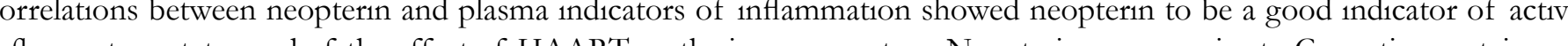

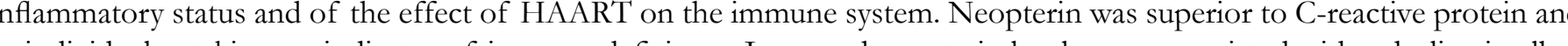
to individual cytokines as indicator of immune deficiency. Increased neopterin levels were associated with a decline in albumin, haemoglobin and the albumin/globulin ratio, and with increases in red cell distribution width.

Conclusions: Plasma neopterin is a good non-specific biomarker of disease activity in HIV/AIDS patients. It is a good indicator of inflammatory activity, perpetuation of inflammation-associated co-morbidities, degree of immune deficiency and has predictive value for underlying disease, and for monitoring the HAART response.

Keywords: neopterin; biomarkers; immune activation; HIV/AIDS

DOI: http://dx.doi.org/10.4314/ahs.v15i2.5

\section{Introduction}

In the present study the suitability of neopterin as a ongoing. Evermore markers are being evaluated as indi- examined. Neopterin, a catabolic product of the pucators of immune suppression and disease progression rine nucleotide guanosine triphosphate, is produced and to better understand the immunopathogenesis of from guanosine 5'-triphosphate (GTP) that is cleaved the disease ${ }^{1,2}$. In contrast to the need for biomark- by GTP- cyclohydrolase 1 to 7,8-dihydroneopterin ers for specific purposes, a general non-specific marker triphosphate, followed by conversion of 7,8-dihydroof disease that could alert the clinician to further in- neopterin triphosphate to neopterin and 7,8-dihydroneopterin under the influence of phosphatases ${ }^{3}$ GTP-cyresource limited environments. Such a tool should be clohydrolase 1 is stimulated, predominantly, by T-helpe relatively inexpensive and facilities for its determination cell type- 1 derived interferon- $\gamma$ but co-stimulation by readily available.

\section{Corresponding author:}

Margaretha Viljoen

Department of Psychiatry;

School of Medicine; Faculty of

Health Sciences; University of Pretoria;

Private Bag X323, 0007, South Africa

Tel: +27012329 3090

Email: mviljoen@webafrica.org.za neopterin levels were compared to that of a number of factors routinely measured for diagnostic purposes and elsewhere described as biomarkers.

\section{Methods}

Study population and ethics statement

This was a cross-sectional, non-intervention study comprising of $105 \mathrm{HIV}$ positive adult patients recruited from the Kalafong secondary hospital in Pretoria, South Africa, and a control group of 60 donors from the South African National Blood Service who tested HIV negative and were therefore considered HIV uninfected. A total of 75 patients were on antiretroviral therapy (HAART group) and 30 patients were HAARTnaïve. Ethical clearance, in accordance with the declaration of Helsinki, the National Health Act and the policy of the University, was received from the Faculty of Health Sciences Research and Ethics Committee of the University of Pretoria (Number: 107/2008). The committee also approved the documentation used for obtaining either written or verbal voluntary informed consent prior to the study. Participants who could not read or write were informed by a clinician about the nature and purpose of the study before verbal consen was obtained. Verbal consent was documented under the relevant section on the consent form which was acknowledged and signed by the clinician and investigator.

\section{Cytokine and neopterin assays}

Plasma neopterin was measured by commercial enzyme

linked immune-absorbent assay (Immuno-Biological Laboratories Inc., USA). The cytokines IL-2, IL-4, IL-

Table 1 Demographic information for the patient and control groups

\begin{tabular}{llll}
\hline & HAART & HAART-naïve & Controls \\
\hline $\mathrm{n}$ & 75 & 30 & 60 \\
Females & $48(64 \%)$ & $18(60 \%)$ & $38(63 \%)$ \\
Age $($ years) & $37.86 \pm 8.86$ & $37.13 \pm 10.24$ & $31.18 \pm 8.09$ \\
BMI $\left(\mathrm{kg} / \mathrm{m}^{2}\right)$ & $23.83 \pm 6.31$ & $20.96 \pm 3.62$ & $21.96 \pm 4.81$ \\
Average months on HAART & $15.86 \pm 16.49$ & - & - \\
TB co-infection at baseline & $14(19 \%)$ & $10(33 \%)$ & - \\
\hline
\end{tabular}

CRP, cytokines and CD4 counts were employed in the IL-6, TNF and IFN- $\gamma$, as well as the anti-inflammatory appraisal of neopterin as non-specific biomarker of the cytokines IL-4 and IL-10. A comparison between the inflammatory status, immune deficiency, the effects of total patient group, the group on HAART, the HAARTanti-retroviral treatment and TB co-infection. The cy- naïve and the control group levels of immunological tokines included the pro-inflammatory cytokines IL-2, factors can be seen in Table 2. 
Table 2 Comparison of immunological and other variables between the controls and patient groups

\begin{tabular}{|c|c|c|c|c|c|c|c|c|}
\hline \multirow[t]{2}{*}{ Variable } & \multirow{2}{*}{$\begin{array}{l}\text { Controls } \\
\text { C }\end{array}$} & \multirow{2}{*}{$\begin{array}{l}\text { Total Patients } \\
T\end{array}$} & \multirow{2}{*}{$\begin{array}{l}\text { HAART } \\
\text { H }\end{array}$} & \multirow{2}{*}{$\begin{array}{l}\text { HAART-Naïve } \\
\mathrm{N}\end{array}$} & \multicolumn{4}{|l|}{ p-value } \\
\hline & & & & & T vs. C & H vs. C & N vs. C & H vs. N \\
\hline $\mathrm{IL}-2(\mathrm{pg} / \mathrm{ml})$ & $9.14 \pm 2.26$ & $20.06 \pm 8.31$ & $18.95 \pm 8.41$ & $22.74 \pm 7.53$ & $<0.0001$ & 0.0003 & $<0.0001$ & 0.076 \\
\hline IL-4 (pg/ml) & $8.07 \pm 2.01$ & $11.96 \pm 4.01$ & $11.65 \pm 4.16$ & $12.70 \pm 3.40$ & $<0.0001$ & $<0.0001$ & $<0.0001$ & 0.198 \\
\hline IL-6 (pg/ml) & $0.69 \pm 1.62$ & $11.16 \pm 14.95$ & $9.56 \pm 12.54$ & $15.04 \pm 19.34$ & 0.0001 & 0.035 & 0.001 & $0.010^{*}$ \\
\hline $\mathrm{IL}-10$ (pg/ml) & $1.45 \pm 1.32$ & $14.61 \pm 12.53$ & $12.44 \pm 12.38$ & $19.82 \pm 11.51$ & $<0.0001$ & $<0.0001$ & $<0.0001$ & $0.026^{*}$ \\
\hline TNF (pg/ml) & $1.71 \pm 1.78$ & $5.74 \pm 3.68$ & $5.65 \pm 3.89$ & $5.95 \pm 3.19$ & $<0.0001$ & 0.0002 & $<0.0001$ & 0.473 \\
\hline IFN- $-(\mathrm{pg} / \mathrm{ml})$ & $24.85 \pm 2.96$ & $44.00 \pm 22.55$ & $41.43 \pm 14.14$ & $53.68 \pm 34.39$ & $<0.0001$ & 0.0003 & $<0.0001$ & $0.017^{*}$ \\
\hline NPT (nmol/l) & $8.23 \pm 5.71$ & $45.57 \pm 41.82$ & $34.51 \pm 35.70$ & $66.63 \pm 40.73$ & $<0.0001$ & $<0.0001$ & $<0.0001$ & $0.0001^{*}$ \\
\hline NPT/LL-4 & $1.04 \pm 0.75$ & $3.81 \pm 3.74$ & $3.04 \pm 3.34$ & $5.63 \pm 4.05$ & 0.001 & 0.02 & 0.0001 & $0.002 *$ \\
\hline IL-2/L-4 & $1.15 \pm 0.26$ & $1.68 \pm 0.76$ & $1.59 \pm 0.64$ & $1.90 \pm 0.97$ & $<0.0001$ & 0.001 & 0.0007 & 0.058 \\
\hline IL-6/L-4 & $0.06 \pm 0.14$ & $0.86 \pm 1.17$ & $0.74 \pm 1.05$ & $1.16 \pm 1.39$ & $<0.0001$ & 0.001 & 0.0065 & 0.102 \\
\hline IFN- $/ / \mathrm{LL}-4$ & $3.25 \pm 1.02$ & $3.97 \pm 3.01$ & $3.59 \pm 1.26$ & $4.87 \pm 5.12$ & 0.030 & 0.019 & 0.009 & 0.050 \\
\hline $\operatorname{CRP}(\mathrm{mg} / \mathrm{l})$ & - & $25.93 \pm 51.23$ & $22.88 \pm 36.13$ & $34.08 \pm 75.78$ & - & - & - & 0.839 \\
\hline 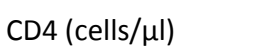 & - & $257.97 \pm 193.06$ & $296.21 \pm 195.50$ & $170.05 \pm 167.26$ & - & - & - & $0.003^{*}$ \\
\hline VL $\left(\log _{10}\right.$ copies $\left./ \mathrm{ml}\right)$ & - & $2.75 \pm 1.36$ & $2.48 \pm 1.12$ & $3.57 \pm 1.71$ & - & - & - & $0.014^{*}$ \\
\hline Albumin $(\mathrm{g} / \mathrm{l})$ & - & $33.40 \pm 7.55$ & $34.94 \pm 6.67$ & $29.50 \pm 8.37$ & - & - & - & $0.027^{*}$ \\
\hline$A / G$ ratio & - & $0.68 \pm 0.25$ & $0.73 \pm 0.24$ & $0.53 \pm 0.21$ & - & - & - & $0.004^{*}$ \\
\hline Haemoglobin $(\mathrm{g} / \mathrm{dl})$ & - & $11.83 \pm 2.36$ & $12.16 \pm 2.36$ & $10.97 \pm 2.05$ & - & - & - & 0.125 \\
\hline RDW (\%) & - & $17.45 \pm 4.35$ & $17.52 \pm 4.50$ & $17.28 \pm 4.01$ & - & - & - & 0.334 \\
\hline
\end{tabular}

Data expressed as mean \pm SD; IL = Interleukin; $T$ NNF = tumor necrosis factor; IFN = interferon; $N P T=$ neopterin; VL = HIV viral load; $\mathrm{A} / \mathrm{G}$ = albumin to globulin ratio; $\mathrm{RDW}=$ red cell distribution width ${ }^{*} p<0.05$ for HAART vs. HAART-naive

The validity of neopterin as indicator of immune de- ficiency was tested against CD4 counts, CRP and cytokine levels. The results can be seen in Table 3 .

Table 3 Correlations of neopterin with CD4 counts, CRP, IL-6, albumin, A/G ratio, haemoglobin and red cell distribution width

\begin{tabular}{lllllll} 
& \multicolumn{2}{l}{ Total Patients } & \multicolumn{2}{l}{ HAART } & \multicolumn{2}{l}{ HAART-Naïve } \\
\cline { 2 - 7 } Neopterin with: & Rho & p-value & Rho & p-value & Rho & p-value \\
\hline CD4 & -0.484 & $0.0001^{*}$ & -0.43 & $0.001^{*}$ & -0.503 & $0.02^{*}$ \\
IL-6 & 0.371 & $0.0010^{*}$ & 0.490 & $0.00001^{*}$ & 0.128 & 0.510 \\
CRP & 0.355 & $0.0006^{*}$ & 0.610 & $0.00001^{*}$ & 0.180 & 0.597 \\
IFN- $y$ & 0.301 & $0.002^{*}$ & 0.277 & $0.017^{*}$ & 0.216 & 0.260 \\
Albumin & -0.547 & $0.0001^{*}$ & -0.447 & $0.0002^{*}$ & -0.457 & $0.014^{*}$ \\
A/G ratio & -0.489 & $0.00001^{*}$ & -0.423 & $0.0004^{*}$ & -0.373 & 0.061 \\
Haemoglobin & -0.597 & $0.00001^{*}$ & -0.555 & $0.00001^{*}$ & -0.33 & 0.093 \\
RDW & 0.342 & $0.001^{*}$ & 0.472 & $0.0001^{*}$ & 0.112 & 0.577 \\
\hline *Spearman Rho rank & correlation statistically significant, & 0.05. IL $=$ interleukin CRP $=$ C-reactive
\end{tabular}

protein; IFN = interferon; $A / G$ ratio = albumin/globulin ratio; RDW = red cell distribution width

The power of discrimination, in terms of area under CD4 counts of less than 200 using logistic regression. the ROC curve (AUROC), of neopterin, CRP and IL-6 The discriminatory power of neopterin (AUROC = was also tested for the total patient group in relation to 0.803 ) was found to be higher than that for CRP (AU ROC $=0.658)$, and for IL-6 (AUROC $=0.753)$
Twenty four of the total HIV patient group were con- for the TB-co-infection group. Likewise neopterin firmed as having tuberculosis $(\mathrm{TB})$ co-infection (spu- $\quad$ (AUROC $=0.7367$ ) showed to be a better discriminata smears). Neopterin $(\mathrm{p}=0.008)$ and CRP $(\mathrm{p}=0.004)$ tor than CRP (AUROC $=0.5945)$ for the TB-negative levels were both significantly higher in the HIV/TB patients.

positive group (neopterin: $65.73 \pm 48.94 \mathrm{nmol} / \mathrm{l}$; CRP: Substances routinely measured in the clinic and previ$47.35 \pm 57.12 \mathrm{mg} / \mathrm{l})$ than in the HIV/TB negative group ously described as biomarkers, i.e., albumin, the albu(neopterin: $37.37 \pm 34.48 \mathrm{nmol} / \mathrm{l}$; CRP: $18.99 \pm 47.56 \mathrm{~min} /$ globulin $(\mathrm{A} / \mathrm{G})$ ratio, haemoglobin and red cell $\mathrm{mg} / \mathrm{l})$. $\quad$ distribution width $(\mathrm{RDW})$ were evaluated as indicators The discriminatory power for neopterin (AUROC $=$ of immune deficiency by comparing their levels to $\mathrm{CD} 4$ 0.898) was higher than that for CRP (AUROC $=0.6252$ ) counts. The results can be seen in Table 4.

Table 4 Correlations for CD4 with blood variables of the different groups

\begin{tabular}{lllllll}
\hline \multirow{2}{*}{ CD4 with: } & \multicolumn{2}{l}{ Total Patients } & \multicolumn{3}{l}{ HAART } & Naïve \\
\cline { 2 - 7 } & Rho & p-value & Rho & p-value & Rho & p-value \\
\hline IL-6 & -0.431 & $0.0001^{*}$ & -0.553 & $0.009^{*}$ & -0.285 & 0.193 \\
CRP & -0.328 & $0.007^{*}$ & -0.410 & $0.005^{*}$ & -0.250 & 0.287 \\
Albumin & 0.491 & $0.00001^{*}$ & 0.497 & $0.0004^{*}$ & 0.142 & 0.551 \\
A/G ratio & 0.486 & $0.00001^{*}$ & 0.505 & $0.0003^{*}$ & 0.113 & 0.636 \\
Haemoglobin & 0.420 & $0.0003^{*}$ & 0.392 & $0.004^{*}$ & 0.256 & 0.276 \\
RDW & -0.370 & $0.010^{*}$ & -0.470 & $0.0004^{*}$ & 0.017 & 0.942 \\
\hline *Spearman Rho rank correlation statistically significant, $\mathrm{p}<0.05$. IL $=$ interleukin; CRP $=$ C-reactive
\end{tabular}

Spearman Rho rank correlation statistically significant, $\mathrm{p}<0.05 . \mathrm{IL}=$ interleukin; CRP $=\mathrm{C}$-reactive protein; $\mathrm{A} / \mathrm{G}$ ratio = albumin/globulin ratio; $\mathrm{RDW}=$ red cell distribution width

Logistic regression results in a comparison between anti-inflammatory, cytokines may be raised ${ }^{5}$, advanced neopterin, albumin, the AG/ratio, haemoglobin and HIV/AIDS is predominantly associated with increased $\mathrm{RDW}$ as indicators of immune deficiency were: neop- inflammatory activity ${ }^{6,7}$. The inflammatory process is terin $($ AUROC $=0.803)$, albumin $($ AUROC $=0.487)$, implicated as a major contributor to the pathogenesis the $\mathrm{A} / \mathrm{G}$ ratio $(\mathrm{AUROC}=0.504)$, haemoglobin $(\mathrm{AU}-$ of many physical disorders, and is likely to play a role ROC $=0.334)$ and RDW $($ AUROC $=0.589)$.

of many physical disorders, and is likely to play a role
in the cardiovascular, renal, liver, metabolic, haematological and skeletal abnormalities, as well as the premature systemic aging associated with HIV infection ${ }^{6,7}$

\section{Discussion}

Increased inflammatory activity is similarly linked to

The value of neopterin as non-specific indicator was neuropsychological impairments ${ }^{8}$, including that found examined in terms of its potential as indicator of in- in HIV/AIDS 9,10 .

flammatory status, the effects of anti-retroviral treat-

ment, as indicator of immune deficiency or dysfunction Neopterin levels are generally considered as an indicaand as indicator of TB co-infection. The validity of ne- tion of both macrophage function and cell mediated compared to that of CRP, cytolines and to a number of substances routinely measured in the clinic. inmunity It is said that when cell-mediated immunity dominates, circulating neopterin levels are usually high and when humoral immunity dominates, neopterin levels are $\operatorname{low}^{3-11}$. Abundant evidence exists for neopterin Neopterin as indicator of inflammatory (cellular levels to be increased in disturbances marked by in immunity) status in HIV AIDS

flammatory activity and raised neopterin levels and inAdvanced HIV/AIDS is characterised by chronic im- creases with disease progression, have previously been mune activation and a concomitant immune deficien- described $^{12}$. However, other substances, in particular cy. Although the levels of pro-inflammatory, as well as CRP and IL-6, have also been described as markers of inflammation. 
In the present study the value of neopterin as general marker of the inflammatory status was examined by comparing neopterin levels to that of two other recognised biomarkers of inflammation, i.e., CRP and IL-6 $6^{6,11-13}$. As seen in Table 2, the mean neopterin levels were significantly higher than that of the control group for both the HAART-naïve $(\mathrm{p}<0.001)$ and the HAART $(p<0.001)$ groups. IL-6 levels were also significantly higher than that of the control group for the HAART naïve $(\mathrm{p}=0.001)$ and the HAART groups $(\mathrm{p}=0.035)$. CRP and IL-6 levels correlated positively with that of neopterin for the total patient group (CRP: $r=0.355, p=0.0006$; IL- $6: \mathrm{r}=0.371, \mathrm{p}=0.001)$ and for the HAART group (CRP: $\mathrm{r}=0.61, \mathrm{p}<0.00001$; IL-6: $\mathrm{r}=0.49, \mathrm{p}<0.0001$ ) (Table 3). These results supported the notion of neopterin as the better marker of inflammatory activity and are in agreement with the concept of advanced HIV/AIDS as a condition with increased inflammatory activity ${ }^{6,7}$

However, although higher pro-inflammatory activity was confirmed in the patients, higher than control levels $(\mathrm{p}<0.05)$ were, as reported elsewhere ${ }^{5}$, also seen in the levels of the anti-inflammatory cytokines (Table 2). Nevertheless, when the pro- versus anti-inflammatory activity was investigated it was found that the ratio neopterin/IL-4 was indeed higher in the HAART-naïve $(\mathrm{p}=0.0001)$, as well as in the HAART patients $(\mathrm{p}=0.02)$, than in the controls. This imbalance with a shift towards inflammation in HIV/AIDS was confirmed by to that of the anti-inflammatory cytokine IL-4. Significantly higher than control ratios were seen for IL-2/IL4 (HAART: $\mathrm{p}=0.001$; HAART-naïve: $\mathrm{p}=0.0007$ ), IL-6/ IL4 (HAART: $\mathrm{p}=0.001$; HAART-naïve: $\mathrm{p}=0.0065$ ), as well as for IFN/IL4 (HAART: $\mathrm{p}=0.019$; HAARTnaïve: $\mathrm{p}=0.009$ )

Pro-inflammatory dominance with cell-mediated immunity in ascendancy was thus shown in the group on anti-retroviral therapy, as well as in the group not yet on anti-retroviral therapy. Such inflammatory activity, demonstrated here by neopterin levels and confirmed by cytokine results, is implicated in the pathogenesis of $\mathrm{HIV} / \mathrm{AIDS}^{6,7}$

Effect of HAART on the pro-inflammatory/anThe mean neopterin level in the HAART group was $48 \%$ lower $(\mathrm{p}=0.0001)$ than that of the HAART-naïve the ratios of the individual pro-inflammatory cytokines i-inflammatory (cellular/humoral immune) statu oup, the mean IL-6 level was $43 \%$ lower $(\mathrm{p}=0.01)$, the mean IFN- $\gamma$ was $22.8 \%$ lower $(\mathrm{p}=0.017$ ), the mean IL-2 was $16.7 \%$ lower $(\mathrm{p}=0.076)$ and the CRP level, although not statistically significant, was 33\% lower. However the HAART group still showed significantly higher in flammatory activity than the control group. In contrast, the levels of the anti-inflammatory cytokine, IL-4, were not significantly different between the HAART and HAART-naïve groups (Table 2). These results, which demonstrated a down-regulation of neopterin and therefore in inflammatory activity upon treatment with highly active antiretroviral treatment are in agreement with that of Amirayan-Chevillard et $1^{14}$. Amirayanvillard et al further showed that neopterin levels again increase with cessation of antiretroviral treatment ${ }^{14}$, a finding that implies neopterin to be a useful marker of the efficacy of HAART and perhaps for the assessment of patient compliance.

As an imbalance between pro- and anti-inflammatory activity, in favour of pro-inflammatory, has previously been implicated in the pathogenesis of HIV/AIDS ${ }^{7}$, it was of interest to see to what extent this imbalance was corrected by antiretroviral treatment. The ratio between neopterin and the main anti-inflammatory cytokine IL-4 for HAART was significantly lower $(\mathrm{p}=0.002)$ than that for the HAART-naive patients - pointing to suppression of cellular (pro-inflammatory) relative to humoral (anti-inflammatory) activity by antiretroviral treatment. However, the ratio in the HAART group was still higher $(\mathrm{p}=0.02)$ than in the control group, confirming the persistence of a shift towards pro-inflammator activity despite anti-retroviral treatment (Table 2). Although the ratios of all the individual pro-inflammatory cytokines to the level of the anti-inflammatory cytokine IL-4 (IL-2/IL-4, IL-6/IL-4, IFN- $\gamma /$ IL-4) were also lower in the HAART than in the HAART-naïve group none were significantly lower.

This persistent, albeit downgraded, presence of inflammatory, and imbalance between pro- and anti-inflammatory activity, despite effective antiretroviral therapy, has previously been reported and is described as a maor contributor to the perpetuation of non-AIDS defining co-morbidities and premature systemic aging in patients on $\mathrm{HAART}^{7}$. Among the best known contributors to such persistence are said to be clinical or subclinical infections, gastrointestinal microbial translocation, infectious, as well as non-infectious, HIV virions and thymic dysfunction ${ }^{7}$
Neopterin as indicator of immune deficiency Neopterin has previously been shown as a measure of the degree of immune deficiency in HIV/AIDS patients ${ }^{2,15,16}$. In the present study the potential of neopterin as indicator of immune deficiency was again investigated by comparing neopterin levels to that of $\mathrm{CD} 4$ counts. Results with neopterin were then compared to that with CRP and cytokines.

Negative correlations were found between neopter$(\mathrm{r}=-0.484, \mathrm{p}=0.0001)$, the HAART group $(\mathrm{r}=-0.43$, $\mathrm{p}=0.001)$ and the HAART-naïve group $(\mathrm{r}=-0.503$, $\mathrm{p}=0.02$ ) (Table 3). 93.3\% of the HAART-naïve patients and $73.3 \%$ of the HAART patients had higher than normal neopterin levels. These results, which suggest neopterin as an indicator of immune deficiency, are in line with that of previous publications $2,15,16$.

Neopterin was subsequently compared to CRP and cytokines as immune deficiency indicators. CRP and one of the cytokines investigated, that is, IL-6, showed significant negative correlations with $\mathrm{CD} 4$ counts for the total patient group (CRP: $r=-0.328, p=0.007$; IL-6: $\mathrm{r}=0.431, \mathrm{p}=0.0001)$ and the HAART group (CRP: $\mathrm{r}=$ the HAART-naïve group (Table 4). In contrast to neopterin, only $49.3 \%$ of the HAART-naïve patients and $46.7 \%$ of the HAART patients had higher than normal CRP reference levels. $83.3 \%$ of the HAART-naive patients and $65.3 \%$ of the HAART patients had higher than normal IL-6 levels.

Logistic regression showed the discriminatory power of neopterin (AUROC $=0.803$ ) to be higher than that for CRP (AUROC $=0.658$ ), and for IL-6 (AUROC $=$ 0.753). Neopterin was therefore shown to be superior to CRP and to individual cytokines as indicator of immune deficiency.

\section{Neopterin and HIV/AIDS with TB-co infection}

Neopterin levels are known to be significantly highe in individuals with active tuberculosis than in patients with inactive tuberculosis or controls ${ }^{17}$. It would thus seem feasible to expect that HIV/AIDS patients with TB-co-infection would have higher levels of neopterin than HIV patients without TB-co-infection. The ability of neopterin to discriminate between HIV patients with and without active TB was therefore investigated and compared to that of CRP and cytokines. in levels and CD4 counts for the total patient group $0.410, \mathrm{p}=0.005$; IL- $-\mathrm{r}: \mathrm{r}=-0.553, \mathrm{p}=0.009)$, but not for

eopterin $(\mathrm{p}=0.008)$ and $\mathrm{CRP}(\mathrm{p}=0.004)$ levels were both significantly higher in the $24 \mathrm{HIV} / \mathrm{TB}$ positive than in the HIV/TB negative patients. This is in agreement with previous reports? ${ }^{9}$. Logistic regression analysis showed the discriminatory power for neopterin (AUROC $=0.898)$ to be higher than that for CRP AUROC $=0.6252$ ). Neopterin therefore seems to be the better indicator of TB-co-infection in patients with HIV/AIDS.

Previous indications that cytokines such as IFN- $\gamma$ TNF-alpha, IL-6, IL-10 and the IFN/IL-10 ratio may be of diagnostic/prognostic value in $\mathrm{TB}^{19-24}$ were not substantiated by the results of the present study. In contrast to our expectations that the balance between pro- and anti-inflammatory activity would be more unavourable with TB-co-infection, the difference for the neopterin/IL-4 ratio between patients with and without TB co-infection $(\mathrm{p}=0.059)$ was non-significant when judged at a 0.05 level of significance.

Advantage of neopterin above that of the measurement of individual cytokines

Locally produced cytokines could bind to their target tissues or be neutralized by soluble receptors ${ }^{25}$. Cy tokine release may therefore not be accurately reflected by circulating levels. The circulating level of neopterin, on the other hand, is largely a product of the balance between synthesis and renal excretion ${ }^{15}$. It therefore speaks for itself that neopterin could very well be better biomarker than individual cytokines. In addition, because of the pleiotropic nature of cytokines as well as the multiple interactions and co-operations between $\mathrm{cy}$ tokines, the measurement of one specific cytokine may not reflect its contribution to inflammation and other immune processes. Neopterin is said to reflect the multiple cooperations between immunocompetent cells ${ }^{26}$.

Comparison of neopterin levels to that of circulating substances and ratios elsewhere described as biomarkers in HIV/AIDS patients

Albumin concentration, the albumin/globulin (A/G) ratio, red cell distribution width (RDW) and haemoglobin $(\mathrm{Hb})$ concentration are routinely measured during clinical investigations. Yet they have elsewhere all been described as biomarkers of disease progression and/or immune deficiency, in general, as well as in HIV/AIDS 7-32. 
Previous associations shown between immune deficien- the total patient group $(\mathrm{r}=0.342, \mathrm{p}=0.001)$ and for the cy (decreased CD4 counts) with albumin, A/G ratios, HAART group $(\mathrm{r}=0.472, \mathrm{p}=0.0001)$. In line with previhaemoglobin and RDW, respectively ${ }^{27-32}$, were support- ous publications, the present study thus demonstrated ed by results on the total patient and HAART groups of a decline in the levels of albumin, the $\mathrm{A} / \mathrm{G}$ ratio and the present study (Table 4). Positive correlations were haemoglobin concentrations, and an increased RDW, found between albumin and the CD4 counts for the with increases in inflammatory activity, as reflected by total patient group $(\mathrm{r}=0.491, \mathrm{p}=0.00001)$ and the group neopterin levels.

on HAART ( $\mathrm{r}=0.497, \mathrm{p}=0.0004)$. Positive contelations were also found between the $\mathrm{A} / \mathrm{G}$ ratio and the $\mathrm{CD} 4$ counts for the total patient group $(\mathrm{r}=0.486, \mathrm{p}=0.00001)$ and the group on HAART $(\mathrm{r}=0.505, \mathrm{p}=0.0003)$. Haemoglobin concentration correlated positively with the CD4 count for the total patient group $(\mathrm{r}=0.42, \mathrm{p}=0.0003)$ and for the HAART group $(\mathrm{r}=0.392, \mathrm{p}=0.004)$. RDW correlated negatively with the $\mathrm{CD} 4$ count for the to-
tal patient group $(\mathrm{r}=-0.37, \mathrm{p}=0.010)$ and the group on HAART $(\mathrm{r}=-0.47, \mathrm{p}=0.0004)$

It is known that the levels of albumin, $A / G$ ratios, haemoglobin and RDW are all adversely influenced by inflammation. With chronic inflammation the levels of albumin, a negative acute-phase protein, decreases as a result of lower synthesis, an increase in fractional metabolic rate, appetite suppression and through an increase in microvascular albumin leakage ${ }^{33,34}$. Chronic inflammatory conditions can influence haemoglobin levels, $\mathrm{RDW}$ and other red blood cell parameters in various ways, most notably processes involved in the anaemia of chronic disease $\mathrm{e}^{35}$. The association between RDW and inflammation is so strong that RDW has been described as indicative of inflammation ${ }^{36}$.

In the present study negative correlations between neopterin and albumin concentrations were observed for the total patient group $(\mathrm{r}=-0.547, \mathrm{p}=00001)$, the group on HAART $(\mathrm{r}=-0.447, \mathrm{p}=0.0002)$ and the HAARTnaïve group ( $\mathrm{r}=-0.475, \mathrm{p}=0.014)$. As for albumin, the $\mathrm{A} / \mathrm{G}$ ratio declined with increases in neopterin as seen in the negative correlations for the total patient group $(\mathrm{r}=-0.489, \mathrm{p}=0.00001)$, the HAART group $(\mathrm{r}=-0.423$, $\mathrm{p}=0.0004)$ and the HAART-naive group $(\mathrm{r}=-0.373$, $\mathrm{p}=0.061)$. Negative correlation were also found between the haemoglobin and neopterin levels for the total patient group $(\mathrm{r}=-0.597, \mathrm{p}=0.00001)$ and for the HAART group ( $\mathrm{r}=-0.555, \mathrm{p}=0.00001)$, while for the HAART-naïve group only a weak negative correlation was found $(r=-0.33, p=0.093)$. Significant positive correlations were found between RDW and neopterin for
This study in HIV/AIDS patients showed neopterin to be a better indicator of the inflammatory status, immune deficiency, TB-co-infection and the effects of HAART than CRP or cytokines. Changes in plasma neopterin levels as an indication of the efficacy of HAART or of patient compliance, should be investigated further In line with the effects of inflammation on various systems, neopterin levels reflect the negative effects of the disease on levels of albumin, haemoglobin, the albu$\mathrm{min} /$ globulin ratio and the red cell distribution width.

It is our contention that neopterin levels represent good non-specific indicator, not only of inflammatory activity, but of the general health status. This statement is made in view of its associations with inflammation, the effects of HAART, immune deficiency, TB-co-infection, the levels of several plasma proteins and, as previously reported, disease progression. The statement is further supported by previously supported diagnostic and prognostic associations between plasma neopterin levels and disorders other than HIV/AIDS. These include viral, intracellular bacterial and parasitic infections, burns, cancer, cardiovascular disease, neurodegeneration, graft versus host disease, autoimmune disorders and a variety of oral afflictions ${ }^{12}$. However, in view of the effect of inflammation on all physiological systems, the primary importance of neopterin probably lies in its reflection of the degree of inflammation, a major contributor to the pathogenesis of HIV/AIDS and a process involved in a wide variety of pathological processes. The skills required for the analysis of neopterin fall within that of most trained laboratory analysts and the costs are below that of the more specialized techniques. Neopterin therefore offers a relatively inexpensive non-specific biomarker in resource limited environments to alert the clinician to investigate further.

\section{Conflict of interest}

None

\section{Acknowledgements:}

We thank the staff and patients of the immunology clinic at Kalafong Hospital in Pretoria, South Africa. We also thank the volunteers and staff of the South African National Blood Service at the Pretoria West satellite site. This research was supported by grant funding received from the Medical Research Council of South African and the South African Sugar Association.

\section{Refences}

- Poli G. Old and new plasma biomarkers in HIV-1 infected African-American women. AIDS. 2011;25(15):1921-1923

2. Bipath P, Viljoen M, Levay PF. Levels of procalcitonin, C-reactive protein and neopterin in patients with advanced HIV-1 infection. S Afr J HIV Med. 2012;13(2):78-82.

3. Murr C, Widner B, Wirleitner B, et al. Neopterin as a marker for immune system activation. Curr Drug Metab. 2002;3(2):175-187.

4. Weiss G, Murr C, Zoller H, et al. Modulation of neopterin formation and tryptophan degradation by Th-1

and Th-2 derived cytokines in human monocyte cells. Clin Exp Immunol. 1999;116:435-440.

5. Shebl F, Yu K, Landgren O, et al. Increased levels of circulating cytokines with HIV-related immunosuppression. AIDS Res Hum Retrovir. 2012;28(8):809-815.

6. Nixon DE, Landay AL. Biomarkers of immune dysfunction in HIV. Curr Opin HIV AIDS. 2010;5(6):498503.

. Desai S, Landay A. Early immune senescence in HIV disease. Curr HIV / AIDS Rep. 2010;7:4-10

8. Viljoen M. Psychoneuroimmunology in terms of the two main stress axes: sickness behaviour as trigger for the development of mental disorders. Thesis (Ph.D), University of Pretoria; 2003

9. Fessel WJ. Impaired neurocognition in HIV infected patients: antecedents and treatment. AIDS 2009;23:1731-1733.

0. Karlsen NR, Frøland SS, Reinvang I. HIV-related neuropsychological impairment and immunodeficiency. CD8+ lymphocytes and neopterin are related to HIV-encephalopathy. Scand J Psychol. 1994;35(3):230239.

11. Koorts AM, Levay PF, Hall AN, et al. Expression of the $\mathrm{H}$-subunit and L-subunit of ferritin in bone marrow macrophages and cells of the erythron during cellular immune activation. Blood Cells Mol Dis. 2011;47:50-55.

12. Plata-Nazar K, Jankowska A. Clinical usefulness of determining the concentration of neopterin. Pteridines. 2011;22:77-89.

13. Shaw AC. Serum C-reactive protein and neopterin concentrations in patients with viral or bacterial infection. J Clin Pathol. 1991;41:596-599.

14. Amirayan-Chevillard N, Tissot-Dupont H, Capo C, et al. Impact of highly active anti-retroviral therapy (HAART) on cytokine production and monocyte subsets in HIV-infected patients. Clin Exp Immunol. 2000;120(1):107-112. 
15. Fuchs D, Weiss G, Wachter H. Neopterin, biochem- 29. Shah S, Smith CJ, Lampe F, et al. Haemoglobin (b) ieactions. Int Arch Alleg Immul 1993;101:-6.

16. Mildvan D, Spritzler J, Grossberg SE, et al. Serum neopterin, an immune activation marker, independently predicts disease progression in advanced HIV-1 infection. Clin Infect Dis. 2005;40(6):853-858. the highly active antiretroviral therapy era: relationships with gender. HIV Med. 2007;8(1):38-45

30. Owiredu WKBU, Quaye L, Amidu N, Addai-Mensah O. Prevalence of anaemia and immunological markers among Ghanaian HAART-naïve HIV-patients and 17. Turout T, Akbulut H, Deveci F, et al. Serum in- those on HAART. African Health Sciences 2011;11(1):2 terleukin-2 and neopterin levels as useful markers for 15.

treatment of active pulmonary tuberculosis. Tohoku J 31. Patel KV, Semba RD, Ferrucci L, et al. Red cell Exp Med. 2006;209(4):321-328.

18. Immanuel C, Victor L, Chelvi KS, et al. Serum ne- ta-analysis. J Gerontol A Biol Sci Med Sci. 2009;65(3):258opterin levels in HIV infected patients with \& without 265

tuberculosis. Ind J Med Res. 2005;121:220-225. tions in IL-10, TNF- $\alpha$, and IFN- $\gamma$ from the earliest AIDS Res Ther. 2007;4:11-25.

point of HIV type 1 infection. AIDS Res Hum Retrovir. 33. Ritchie RF, Palomaki GE, Neveux LM, et al. Ref2006;22(8):757-762.

20. Orsilles MA, Pieri E, Cooke P, et al. IL-2 and IL-10 serum levels in HIV-1-infected patients with or without active antiretroviral therapy. APMIS. 2006;114:55-60. 21. Ullum H, Diamant M, Victor J, et al. Increased circulating levels of interleukin-6 in HIV-seropositive subjects. Acq Imm Def Synd. 1996;13(1):93-94.

22. Breen EC, Rezai AR, Nakajima K, et al. Infection with HIV is associated with elevated IL-6 levels and production. J Immunol. 1990;144(2):480-484.

23. Stylianou E, Aukrust P, Kvale D, et al. IL-10 in HIV infection: increasing serum IL-10 levels with disease progression - down-regulatory effect of potent anti-retroviral therapy. Clin Exp Immunol. 1999;116:115-120. 24. Jamil B, Shahid F, Hasan Z, et al. Interferon $\gamma /$ IL10 ratio defines the disease severity in pulmonary and extra pulmonary tuberculosis. Tuberculosis. 2007:87:279-287. 25. Diez-Ruiz A, Tilz GP, Zangerle R, et al. Soluble receptors for tumor necrosis factor in clinical laboratory diagnosis. Eur J Haematol. 1995;54:1-8. proteins, albumin, transferrin, and transthyretin: a practical, simple and clinically relevant approach in a large cohort. J Clin Lab Anal. 1999;13(6):273-279.

34. Don BR, Kaysen G. Serum Albumin: relationship to (nflammation and nutrition. Semin Dial. 2004:17(6):432437.

35. Moyle G. Anaemia in patients with HIV infection: Prognostic marker and contributor to morbidity. AIDS Rev. 2002;4:13-20.

6. Lippi G, Targher G, Montagnana M, et al. Relation between red blood cell distribution width and inflammatory biomarkers in a large cohort of unselected outpatients. Arch Pathol Lab Med. 2009;133(4):628-632. 37. Wirleitner B, Neurauter G, Schröcksnadel K, et al. Interferon-gamma-induced conversion of tryptophan: immunologic and neuropsychiatric aspects. Curr Med Chem. 2003;10(16):1581-1591.

38. Gil L, Martinez G, González I, et al. Contribution to characterization of oxidative stress in HIV/AIDS 26. Wirleitner B, Schroecksnadel K, Winkler C, et al. Ne- patients. Pharmacol Res. 2003;47:217-224.

opterin in HIV-1 infection. Mol Immunol. 2005;42:183- 39. Murr C, Winklhofer-Roob BM, Schroecksnadel K, 194.

27. Mildvan D, CreaghT, Leitz G. Prevalence of anemia and correlation with biomarkers and specific an tiretroviral regimens in 9690 human immunodeficiency virus-infected patients: findings of the Anemia Prevalence Study. Curr Med Res Opinion. 2007;23(2):343-353. 28. Ramana KV, Chary J, Sabitha V, et al. Role of hematological and alternate markers in human immunodeficiency virus disease progression. Am Med J. 2010;1:8487. t al. Inverse association between serum concentration of neopterin and antioxidants in patients with and without angiographic coronary artery disease. Atherosclerosis. 2009;202(2):543-549

40. Widner B, Enzinger C, Laich A, et al. Hyperhomocysteinemia, pteridines and oxidative stress. Curr Drus Metab. 2002;3(2):225-232.

41. Wirleitner B, Bitterlich-Baier G, Hoffman G, et al Neopterin derivatives to activate NF-KB. Free Radical Biol Med. 1997;23:177-178.
42. Überall F, Werner-Felmayer G, Schubert C, et al. 43. Schobersberger W, Hoffmann G, Grote J, et al. InNeopterin derivatives together with cyclic guanosine duction of inducible nitric oxide synthase expression by monophosphate induce c-fos gene expression. FEBS neopterin in vascular smooth muscle cells. FEBS Lett. Lett. 1994;352:11-14. 